\title{
Dual-phase-lag one-dimensional thermo-porous-elasticity with microtemperatures
}

\author{
Z. Liu ${ }^{1} \cdot$ R. Quintanilla ${ }^{2}$ (D)
}

Received: 4 March 2021 / Revised: 30 July 2021 / Accepted: 30 July 2021 /

Published online: 30 August 2021

(c) The Author(s) 2021

\begin{abstract}
This paper is devoted to studying the linear system of partial differential equations modelling a one-dimensional thermo-porous-elastic problem with microtemperatures in the context of the dual-phase-lag heat conduction. Existence, uniqueness, and exponential decay of solutions are proved. Polynomial stability is also obtained in the case that the relaxation parameters satisfy a certain equality. Our arguments are based on the theory of semigroups of linear operators.
\end{abstract}

Keywords Dual phase-lag heat conduction with microtemperatures .

Thermo-porous-elasticity · Microtemperatures · Well-posedness · Exponential stability ·

Polynomial stability $\cdot$ Semigroups

Mathematics Subject Classification 74F05 $\cdot 74 \mathrm{~F} 99 \cdot 74 \mathrm{H} 20 \cdot 74 \mathrm{H} 40 \cdot 74 \mathrm{H} 55$

\section{Introduction}

At the beginning of the last century, Cosserat brothers (Cosserat and Cosserat 1909) proposed the study of the micropolar elastic solids; that is a type of material such that the material points can rotate and, therefore, microstructure is taken into account. In the second part of the last century, there were extensive study about elastic materials with microstructure based on the axioms of thermomechanics. In the work of Goodman and Cowin (1972), the authors proposed the foundations of a continuum theory for granular materials with interstitial voids. Their basic idea consists in writing the bulk density as the product of the density matrix by

Communicated by José R Fernández.

$凶 \quad$ R. Quintanilla

ramon.quintanilla@upc.edu

Z. Liu

zliu@d.umn.edu

1 Department of Mathematics and Statistics, University of Minnesota Duluth, Duluth, MN 55812, USA

2 Departamento de Matemáticas, E.S.E.I.A.A.T.-U.P.C., Colom 11, 08222 Terrassa, Barcelona, Spain 
the volume fraction. Based on this point of view, (Cowin 1985; Cowin and Nunziato 1983; Nunziato and Cowin 1979) set down the theory of elastic solids with voids. The intention was to model the deformations of solids with pores or small voids distributed within them. Thermal effects were also included (Ieşan 1986, 2004; Ieşan and Nappa 2004). It is worth recalling that this theory has received a lots of attention in the past years (Feng and Apalara 2019; Feng and Yin 2019; Leseduarte et al. 2010; Magaña and Quintanilla 2006, 2007; Miranville and Quintanilla 2019, 2020; Pamplona et al. 2011; Santos et al. 2017) to understand the relevance of the microstructural component in the whole material. In fact, the microstructure has deserved much attention and one of its possible components is the microtemperature (Bazarra et al. 2019; Casas and Quintanilla 2005; Feng et al. 2020; Grot 1969; Ieşan 2007; Ieşan and Quintanilla 2000; Magaña and Quintanilla 2018; Passarella et al. 2017; Riha 1975, 1976).

Heat conduction is usually based on the Fourier law. In this case, the heat flux vector is expressed as a linear form of the gradient of temperature. However, this assumption brings us to a paradox, because the thermal waves propagates instantaneously and, therefore, the causality principle is violated. It has been natural to look for an alternative law for the heat flux vector. Cattaneo and Maxwell proposed the introduction of a relaxation parameter which brings to a hyperbolic damped equation. Other authors, such as Green and Naghdi, have proposed alternative laws. In this paper, we recover the one proposed by Tzou (1995). The introduction of two delay parameters is considered, and the Cattaneo and Maxwell law can be seen as a particular case. It is worth recalling that the first contribution concerning the decay of solutions for the dual-phase-lag thermoelasticity was presented by Quintanilla and Racke (2006). Many results dealing this thermoelatic theory have been obtained later. Recently, Liu et al. (2020) proposed the study of the dual-phase-lag heat conduction with microtemperatures and gave sufficient conditions to guarantee the stability of the problem (Borgmeyer et al. 2014; Liu et al. 2017; Liu and Quintanilla 2018; Magaña and Quintanilla 2018).

In this short note, we want to focus our attention to porous-thermo-elastic materials with microtemperatures in the context of the dual-phase-lag theory. That is to consider the elastic materials with voids where the heat conduction and the microheat conduction are determined by the dual-phase-lag theory. In this sense, the present paper develops three main objectives: the first one is to propose the one-dimensional thermo-porous-elasticity with microtemperatures in the context of the dual-phase-lag heat conduction. The second one is to provide of a family of conditions on the parameters of the system guaranteeing the well-posedness of the problem in a suitable Hilbert space. The third one is to prove an exponential stability result for the solutions. We also obtain the polynomial stability of the solutions when the relaxation parameters satisfy a certain relation (limit case).

\section{Basic equations}

This section is devoted to set down the basic equations for the one-dimensional problem of the thermo-porous-elasticity with microtemperatures for isotropic and homogeneous materials in the context of the dual-phase-lag theory. As we consider the case of a rod, we could think that it occupies the interval of length $\pi$. In this case, the evolution equations are

$$
\begin{aligned}
& \rho \ddot{u}=t_{x}, \quad J \ddot{\phi}=h_{x}+g, \\
& \rho T_{0} \dot{\eta}=q_{x}, \quad \rho \dot{\epsilon}=P_{x}+q-Q,
\end{aligned}
$$


and the constitutive equations ${ }^{1}$

$$
\begin{aligned}
& t=\mu u_{x}+\mu_{0} \phi-\beta_{0} \theta, \quad h=a_{0} \phi_{x}-\mu_{2} T, \\
& g=-\mu_{0} u_{x}-\xi \phi+\beta_{1} \theta, \quad \rho \eta=\beta_{0} u_{x}+\beta_{1} \phi+a \theta, \\
& \rho \epsilon=-\mu_{2} \phi_{x}-b T \\
& q+\tau_{1} \dot{q}+\frac{\tau_{1}^{2}}{2} \ddot{q}=\left(k \theta_{x}+k_{1} T\right)+\tau_{2}\left(k \dot{\theta}_{x}+k_{1} \dot{T}\right), \\
& P+\tau_{1} \dot{P}+\frac{\tau_{1}^{2}}{2} \ddot{P}=-k_{4}\left(S_{x}+\tau_{2} \dot{S}_{x}\right), \\
& Q+\tau_{1} \dot{Q}+\frac{\tau_{1}^{2}}{2} \ddot{Q}=\left(k-k_{1}\right) \theta_{x}+\left(k_{1}-k_{2}\right) T+\tau_{2}\left(\left(k-k_{1}\right) \dot{\theta}_{x}+\left(k_{1}-k_{2}\right) \dot{T}\right) .
\end{aligned}
$$

In the above system of equations $\rho$ is the mass density, $u$ is the displacement, $t$ is the stress, $h$ is the equilibrated stress, $g$ is the equilibrated body force, $\eta$ is the entropy, $q$ is the heat flux, $J$ is the equilibrated inertia, $T_{0}$ is the reference temperature at the equilibrium state (that we will assume equal to one), $\epsilon$ is the first moment of the energy, $Q$ is the microheat flux average, $P$ is the first heat flux moment, $\phi$ is the volume fraction, $\theta$ is the temperature, $T$ is the microtemperature, and $\tau_{1}$ and $\tau_{2}$ are the relaxation parameters. The constitutive parameters, $\mu, \mu_{0}, \beta_{0}, \beta_{1}, a_{0}, \mu_{2}, \xi, k$ and $k_{i}$ define the characteristics of the material, and in particular, they define the couplings ${ }^{2}$.

We will assume that

$$
\begin{aligned}
& \mu>0, \quad \mu \xi>\mu_{0}^{2}, \quad a_{0}>0, \quad k>0, \quad k_{4}>0, \quad \rho>0, \\
& J>0, \quad a>0, \quad b>0, \quad k k_{2}>k_{1}^{2}, \quad 2 \tau_{2} \geq \tau_{1}>0 .
\end{aligned}
$$

These assumptions are natural in the context of the theory. In particular, they imply that the internal energy and the dissipation are positive definite bilinear forms which are related to the elastic stability. We also mention that the last condition on the relaxation parameters implies that the heat conduction is stable and dissipative (Liu et al. 2020) (see also the numerical treatment (Bazarra et al. 2021)). When the relaxation parameters do not satisfy this condition, the instability of solutions holds (Quintanilla 2003).

If we substitute the constitutive equations into the evolution equations, we obtain the following linear system:

$$
\begin{aligned}
& \rho \ddot{u}=\mu u_{x x}+\mu_{0} \phi_{x}-\beta_{0} \theta_{x}, \\
& J \ddot{\phi}=a_{0} \phi_{x x}-\mu_{0} u_{x}-\mu_{2} T_{x}+\beta_{1} \theta-\xi \phi, \\
& a\left(\dot{\theta}+\tau_{1} \ddot{\theta}+\frac{\tau_{1}^{2}}{2} \dddot{\theta}\right)=-\beta_{0}\left(\dot{u}_{x}+\tau_{1} \ddot{u}_{x}+\frac{\tau_{1}^{2}}{2} \dddot{u}_{x}\right) \\
& \quad-\beta_{1}\left(\dot{\phi}+\tau_{1} \ddot{\phi}+\frac{\tau_{1}^{2}}{2} \dddot{\phi}\right)+k\left(\theta_{x x}+\tau_{2} \dot{\theta}_{x x}\right)+k_{1}\left(T_{x}+\tau_{2} \dot{T}_{x}\right), \\
& b\left(\dot{T}+\tau_{1} \ddot{T}+\frac{\tau_{1}^{2}}{2} \dddot{T}\right)=-\mu_{2}\left(\dot{\phi}_{x}+\tau_{1} \ddot{\phi}_{x}+\frac{\tau_{1}^{2}}{2} \dddot{\phi}_{x}\right)+k_{4}\left(T_{x x}+\tau_{2} \dot{T}_{x x}\right)
\end{aligned}
$$

\footnotetext{
1 A general formulation of the constitutive equations would allow that the relaxation parameters for the macroscopic structure could be different from the ones corresponding to the microstructure. However, as our contribution try to be a pioneering work in the study concerning dual-phase-lag at the microstructure, we consider the easier case from the mathematical aspect and it corresponds to the case when they agree. The general case is much more difficult from a mathematical point of view and the analysis of the general case is not clear even for the rigid solid.
}

2 We have assumed that the Onsager postulate is satisfied (see Eringen 1999, p.55). 


$$
-k_{2}\left(T+\tau_{2} \dot{T}\right)-k_{1}\left(\theta_{x}+\tau_{2} \dot{\theta}_{x}\right) .
$$

If we denote by $\hat{f}=f+\tau_{1} \dot{f}+\frac{\tau_{1}^{2}}{2} \ddot{f}$, we can write our system as

$$
\begin{aligned}
& \rho \ddot{\hat{u}}=\mu \hat{u}_{x x}+\mu_{0} \hat{\phi}_{x}-\beta_{0}\left(\theta_{x}+\tau_{1} \dot{\theta}_{x}+\frac{\tau_{1}^{2}}{2} \ddot{\theta}_{x}\right), \\
& J \ddot{\hat{\phi}}=a_{0} \hat{\phi}_{x x}-\mu_{0} \hat{u}_{x}-\mu_{2}\left(T_{x}+\tau_{1} \dot{T}_{x}+\frac{\tau_{1}^{2}}{2} \ddot{T}_{x}\right)+\beta_{1}\left(\theta+\tau_{1} \dot{\theta}+\frac{\tau_{1}^{2}}{2} \ddot{\theta}\right)-\xi \hat{\phi}, \\
& a\left(\dot{\theta}+\tau_{1} \ddot{\theta}+\frac{\tau_{1}^{2}}{2} \dddot{\theta}\right)=-\beta_{0} \dot{\hat{\hat{u}}}_{x}-\beta_{1} \dot{\hat{\phi}}+k\left(\theta_{x x}+\tau_{2} \dot{\theta}_{x x}\right)+k_{1}\left(T_{x}+\tau_{2} \dot{T}_{x}\right), \\
& b\left(\dot{T}+\tau_{1} \ddot{T}+\frac{\tau_{1}^{2}}{2} \dddot{T}\right)=-\mu_{2} \dot{\hat{\phi}}_{x}+k_{4}\left(T_{x x}+\tau_{2} \dot{T}_{x x}\right)-k_{2}\left(T+\tau_{2} \dot{T}\right)-k_{1}\left(\theta_{x}+\tau_{2} \dot{\theta}_{x}\right) .
\end{aligned}
$$

From now on, we will omit the hats on the mechanical variables to simplify the notation.

To propose the well-posed problem, we will need to impose the initial conditions

$$
\begin{array}{lll}
u(x, 0)=u^{0}(x), & \dot{u}(x, 0)=v^{0}(x), \quad \phi(x, 0)=\phi^{0}(x), \quad \dot{\phi}(x, 0)=\varphi^{0}(x), \\
\theta(x, 0)=\theta^{0}(x), & T(x, 0)=T^{0}(x), \quad \dot{\theta}(x, 0)=\vartheta(x), \quad \dot{T}(x, 0)=S^{0}(x) \\
\ddot{\theta}(x, 0)=\zeta^{0}(x), & \ddot{T}(x, 0)=R^{0}(x),
\end{array}
$$

where $u^{0}, v^{0}, \phi^{0}, \varphi^{0}, \theta^{0}, \vartheta^{0}, \zeta^{0}, T^{0}, S^{0}$, and $R^{0}$ are given functions.

Since we assume homogeneous Dirichlet boundary conditions, it follows that:

$$
u(x, t)=\phi(x, t)=\theta(x, t)=T(x, t)=0, \quad t \in[0, \infty), \quad x=0, \pi .
$$

In this situation, the energy of the system is given by

$$
\begin{aligned}
E(t)= & \frac{1}{2} \int_{0}^{\pi}\left(\rho|\dot{u}|^{2}+J|\dot{\phi}|^{2}+\mu\left|u_{x}\right|^{2}+2 \mu_{0} u_{x} \phi+\xi|\phi|^{2}+a\left|\phi_{x}\right|^{2}\right) d x \\
& +\frac{1}{2} \int_{0}^{\pi}\left(a|\hat{\theta}|^{2}+b|\hat{T}|^{2}+k\left(\tau_{1}+\tau_{2}\right)\left|\theta_{x}\right|^{2}+\frac{k \tau_{1}^{2} \tau_{2}}{2}\left|\dot{\theta}_{x}\right|^{2}+k_{2}\left(\tau_{1}+\tau_{2}\right)|T|^{2}\right. \\
& \left.+\frac{k_{2} \tau_{1}^{2} \tau_{2}}{2}|\dot{T}|^{2}+k \tau_{1}^{2} \theta_{x} \dot{\theta}_{x}\right) d x \\
& +\frac{1}{2} \int_{0}^{\pi}\left(k_{2} T \dot{T}+2\left(\tau_{1}+\tau_{2}\right) k_{1} \theta_{x} T+k_{1} \tau_{1}^{2}\left(\theta_{x} \dot{T}+\dot{\theta}_{x} T\right)\right. \\
& +k_{1} \tau_{1}^{2} \tau_{2} \dot{\theta}_{x} \dot{T}+k_{4}\left(\tau_{1}+\tau_{2}\right)\left|T_{x}\right|^{2} \\
& \left.+\frac{\tau_{1}^{2}}{2} k_{4}\left|\dot{T}_{x}\right|^{2}+\tau_{1}^{2} k_{4} T_{x} \dot{T}_{x}\right) d x .
\end{aligned}
$$

The dissipation is given by

$$
\begin{aligned}
D(t)= & \int_{0}^{\pi}\left[k\left|\theta_{x}\right|^{2}+k\left(\tau_{1} \tau_{2}-\frac{\tau_{1}^{2}}{2}\right)\left|\dot{\theta}_{x}\right|^{2}+k_{2}|T|^{2}+k_{2}\left(\tau_{1} \tau_{2}-\frac{\tau_{1}^{2}}{2}\right)|\dot{T}|^{2}\right. \\
& \left.+2 k_{1} \theta_{x} T+2 k_{1}\left(\tau_{1} \tau_{2}-\frac{\tau_{1}^{2}}{2}\right) \dot{\theta}_{x} \dot{T}\right] d x \\
& +\int_{0}^{\pi}\left[k_{4}\left|T_{x}\right|^{2}+\left(\tau_{1} \tau_{2}-\frac{\tau_{1}^{2}}{2}\right)\left|\dot{T}_{x}\right|^{2}\right] d x .
\end{aligned}
$$

As we said before, under the assumptions we imposed previously, the energy and the dissipation are positive definite. 
We have

$$
E(t)+\int_{0}^{t} D(\xi) d \xi=E(0) .
$$

Therefore, we can expect the stability of the solutions of the problem. In fact, we will show the exponential stability (or polynomial).

\section{Existence and uniqueness}

This section is devoted to show the well-posedness of the problem proposed previously. Therefore, our intention is to transform our problem into a Cauchy problem in a suitable Hilbert space.

We will propose the problem in the Hilbert space

$$
\mathcal{H}=W_{0}^{1,2} \times L^{2} \times W_{0}^{1,2} \times L^{2} \times W_{0}^{1,2} \times W_{0}^{1,2} \times L^{2} \times W_{0}^{1,2} \times W_{0}^{1,2} \times L^{2} .
$$

As usual, $W_{0}^{1,2}$ and $L^{2}$ are the usual Hilbert spaces. It is worth noting that now we consider that the elements take values in the complex field.

An element in this space will be denoted by $U=(u, v, \phi, \varphi, \theta, \vartheta, \zeta, T, S, R)$.

Defining an operator $\mathcal{A}: \mathcal{H} \rightarrow \mathcal{H}$ by

$$
\mathcal{A} U=\left\{\begin{array}{l}
v \\
\frac{1}{\rho}\left[\mu u_{x}+\mu_{0} \phi-\beta_{0}\left(\theta+\tau_{1} \vartheta+\frac{\tau_{1}^{2}}{2} \zeta\right)\right]_{x} \\
\varphi \\
\frac{1}{J}\left[\left(a_{0} \phi_{x}-\mu_{2}\left(T_{x}+\tau_{1} S_{x}+\frac{\tau_{1}^{2}}{2} R_{x}\right)\right)_{x}-\mu_{0} u_{x}-\xi \phi+\beta_{1}\left(\theta+\tau_{1} \vartheta+\frac{\tau_{1}^{2}}{2} \zeta\right)\right] \\
\zeta \\
\frac{2}{a \tau_{1}^{2}}\left[-\beta_{0} v_{x}-\beta_{1} \varphi+k\left(\theta_{x}+\tau_{2} \vartheta_{x}\right)_{x}+k_{1}\left(T_{x}+\tau_{2} S_{x}\right)-a \vartheta-a \tau_{1} \zeta\right] \\
S \\
\frac{2}{b \tau_{1}^{2}}\left[-\mu_{2} \varphi_{x}+k_{4}\left(T_{x}+\tau_{2} S_{x}\right)_{x}-k_{2}\left(T+\tau_{2} S\right)-k_{1}\left(\theta+\tau_{2} \vartheta_{x}\right)-a S-a \tau_{1} R\right]
\end{array}\right.
$$

with the domain

$$
\mathcal{D}(\mathcal{A})=\left\{U \in \mathcal{H} \mid v, \varphi, \zeta, R \in W_{0}^{1,2}, \quad u_{x x}, \phi_{x x}, \theta_{x x}+\tau_{2} \vartheta_{x x}, T_{x x}+\tau_{2} S_{x x} \in L^{2}\right\} .
$$

We then can write our problem as

$$
\frac{d U}{d t}=\mathcal{A} U, \quad U(0)=U^{0},
$$

where $U^{0}=\left(u^{0}, v^{0}, \phi^{0}, \varphi^{0}, \theta^{0}, \vartheta^{0}, \zeta^{0}, T^{0}, S^{0}, R^{0}\right)$.

The main aim of this section is to prove that the operator $\mathcal{A}$ generates a $C_{0}$ semigroup of contractions on $\mathcal{H}$.

Given $U=(u, v, \phi, \varphi, \theta, \vartheta, \zeta, T, S, R)$ and $U^{*}=\left(u^{*}, v^{*}, \phi^{*}, \varphi^{*}, \theta^{*}, \vartheta^{*}, \zeta^{*}, T^{*}, S^{*}\right.$, $\left.R^{*}\right)$, we consider the inner product defined as

$$
\begin{aligned}
& \left\langle U, U^{*}\right\rangle_{\mathcal{H}}=\frac{1}{2} \int_{0}^{\pi}\left(\rho v \bar{v}^{*}+J \varphi \bar{\varphi}^{*}+\mu u_{x} \bar{u}_{x}^{*}+\mu_{0}\left(u_{x} \bar{\phi}^{*}+\bar{u}_{x}^{*} \phi\right)+\xi \phi \bar{\phi}^{*}\right. \\
& \left.+a_{0} \phi_{x} \bar{\phi}_{x}^{*}+c \theta \overline{\hat{\theta}}^{*}+b T \overline{\hat{T}}^{*}\right) d x
\end{aligned}
$$




$$
\begin{aligned}
& +\frac{1}{2} \int_{0}^{\pi}\left(k\left(\tau_{1}+\tau_{2}\right) \theta_{x} \bar{\theta}_{x}^{*}+\frac{k \tau_{1}^{2} \tau_{2}}{2} \vartheta_{x} \bar{\vartheta}_{x}^{*}+k_{2}\left(\tau_{1}+\tau_{2}\right) T \bar{T}^{*}+\frac{k_{2} \tau_{1}^{2} \tau_{2}}{2} T \bar{T}^{*}\right. \\
& \left.+\frac{k \tau_{1}^{2}}{2}\left(\theta_{x} \bar{\vartheta}_{x}^{*}+\bar{\theta}_{x}^{*} \vartheta_{x}\right)\right) d x \\
& +\frac{1}{2} \int_{0}^{\pi}\left(\frac{k_{2}}{2}\left(T \bar{S}^{*}+\bar{T}^{*} S\right)+k_{1}\left(\tau_{1}+\tau_{2}\right)\left(\theta_{x} \bar{T}^{*}+\bar{\theta}_{x}^{*} T\right)+\frac{k_{1} \tau_{1}^{2} \tau_{2}}{2}\left(\vartheta_{x} \bar{S}^{*}+\bar{\vartheta}_{x}^{*} S\right)\right) d x \\
& +\frac{1}{2} \int_{0}^{\pi}\left(\frac{k_{1} \tau_{1}^{2}}{2}\left(\theta_{x} \bar{S}^{*}+\bar{\theta}_{x}^{*} S\right)+k_{4}\left(\tau_{1}+\tau_{2}\right) T_{x} \bar{T}_{x}^{*}+\frac{k_{4} \tau_{1}^{2}}{2} S_{x} \bar{S}_{x}^{*}\right. \\
& \left.+\frac{k_{4} \tau_{1}^{2}}{2}\left(T_{x} \bar{S}_{x}^{*}+\bar{T}_{x}^{*} S_{x}\right)\right) d x \\
& +\frac{1}{2} \int_{0}^{\pi} \frac{k_{1} \tau_{1}^{2}}{2}\left(\vartheta_{x} \bar{T}^{*}+\bar{\vartheta}_{x}^{*} T\right) d x .
\end{aligned}
$$

Here, and from now on, the bar denotes the conjugated complex. It is clear that this inner product is equivalent to the usual one in the Hilbert space $\mathcal{H}$ (see Liu et al. 2020).

Theorem 1 Assume that the conditions (1)-(2) hold. Then, operator $\mathcal{A}$ generates a $C_{0}$ semigroup of contractions.

Proof It is clear that $\mathcal{D}(\mathcal{A})$ is dense in $\mathcal{H}$. We can easily show that

$$
\begin{aligned}
& \operatorname{Re}\langle\mathcal{A} U, U\rangle_{\mathcal{H}}=-\frac{1}{2} \int_{0}^{\pi}\left[k\left|\theta_{x}\right|^{2}+k\left(\tau_{1} \tau_{2}-\frac{\tau_{1}^{2}}{2}\right)\left|\vartheta_{x}\right|^{2}+k_{2}|T|^{2}\right. \\
& \left.\quad+k_{2}\left(\tau_{1} \tau_{2}-\frac{\tau_{1}^{2}}{2}\right)|S|^{2}+2 k_{1} \operatorname{Re} \theta_{x} \bar{T}\right] d x \\
& \quad-\frac{1}{2} \int_{0}^{\pi}\left[2 k_{1}\left(\tau_{1} \tau_{2}-\frac{\tau_{1}^{2}}{2}\right) \operatorname{Re} \vartheta_{x} \bar{S}+k_{4}\left|T_{x}\right|^{2}+k_{4}\left(\tau_{1} \tau_{2}-\frac{\tau_{1}^{2}}{2}\right)\left|S_{x}\right|^{2}\right] d x .
\end{aligned}
$$

In view of the assumptions we have imposed on the constitutive coefficients, we see that this is less or equal to zero (see Liu et al. 2020).

Now, we prove that zero belongs to the resolvent of the operator. Given $\left(f_{1}, f_{2}, f_{3}, f_{4}, f_{5}\right.$, $\left.f_{6}, f_{7}, f_{8}, f_{9}, f_{10}\right) \in \mathcal{H}$, we must show that the system

$$
\begin{aligned}
& v=f_{1}, \quad \varphi=f_{3}, \quad \vartheta=f_{5}, \quad \zeta=f_{6}, \quad S=f_{8}, \quad R=f_{9} \\
& \mu u_{x x}+\mu_{0} \phi_{x}-\beta_{0}\left(\theta_{x}+\tau_{1} \vartheta_{x}+\frac{\tau_{1}^{2}}{2} \zeta_{x}\right)=\rho f_{2}, \\
& a_{0} \phi_{x x}-\mu_{0} u_{x}-\mu_{2}\left(T_{x}+\tau_{1} S_{x}+\frac{\tau_{1}^{2}}{2} R_{x}\right)+\beta_{1}\left(\theta+\tau_{1} \vartheta+\frac{\tau_{1}^{2}}{2} \zeta\right)-\xi \phi=J f_{4}, \\
& \quad-\beta_{0} v_{x}-\beta_{1} \varphi+k\left(\theta_{x x}+\tau_{2} \vartheta_{x x}\right)+k_{1}\left(T_{x}+\tau_{2} S_{x}\right)-a \vartheta-a \tau_{1} \zeta=\frac{a \tau_{1}^{2}}{2} f_{5}, \\
& -\mu_{2} \varphi_{x}+k_{4}\left(T_{x x}+\tau_{2} S_{x x}\right)-k_{2}\left(T+\tau_{2} S\right)-k_{1}\left(\theta+\tau_{2} \vartheta_{x}\right)-a S-a \tau_{1} R=\frac{b \tau_{1}^{2}}{2} f_{6},
\end{aligned}
$$

has a solution in the domain of operator $\mathcal{A}$. The solution for $v, \varphi, \vartheta, \zeta, S$, and $R$ is directly obtained. Therefore, we obtain the system of equations

$$
\begin{aligned}
& \mu u_{x x}+\mu_{0} \phi_{x}-\beta_{0} \theta_{x}=F_{1}, \\
& a_{0} \phi_{x x}-\mu_{0} u_{x}-\mu_{2} T_{x}+\tau_{1} S_{x}+\beta_{1} \theta-\xi \phi=F_{2},
\end{aligned}
$$




$$
\begin{aligned}
& k \theta_{x x}+k_{1} T_{x}=F_{3} \\
& k_{4} T_{x x}-k_{2} T-k_{1} \theta_{x}=F_{4} .
\end{aligned}
$$

Here, $F_{i}, i=1, \cdots, 4$ can be obtained in terms of the $f_{i}, i=1 \ldots 10$. What it is relevant is that $F_{i}$ belongs to $W^{-1,2}$ for every $i=1 \ldots 4$. If we look to the last two equations, we can define the bilinear form

$$
\mathcal{B}\left[\left(\theta_{1}, T_{1}\right),\left(\theta_{2}, T_{2}\right)\right]=\int_{0}^{\pi}\left(\left(k \theta_{1, x x}+k_{1} T_{1, x}\right) \bar{\theta}_{2}+\left(k_{4} T_{1, x x}-k_{2} T_{1}-k_{1} \theta_{1, x}\right) \bar{T}_{2}\right) d x .
$$

It is bounded and coercive in $W_{0}^{1,2} \times W_{0}^{1,2}$. By the Lax-Milgram lemma, we see the existence of $\theta$ and $T$ satisfying the last two equations. Moreover, they belong to $W_{0}^{1,2}$. Thus, we can substitute them into the first two equations and use again the Lax-Milgram lemma to prove the existence of $u$ and $\phi$ also in $W_{0}^{1,2}$.

In view of the Lumer-Phillips corollary to the Hille-Yosida theorem, we find that our operator generates a $C_{0}$ semigroup of contractions on $\mathcal{H}$.

Thus, we have proved the following result.

Theorem 2 Assume that conditions (1)-(2) hold, then, for every $U^{0} \in \mathcal{D}(\mathcal{A})$, there exists a unique solution to problem (4).

We note that, since the operator generates a contractive semigroup, the problem is stable and well-posed in the sense of Hadamard. Furthermore, in case that we impose supply terms with suitable regularity conditions, the solutions will depend continuously on the supply terms.

\section{Exponential decay: case of $2 \tau_{2}>\tau_{1}$}

The aim of this section is to prove that the solutions of our problem decay in an exponential way to the equilibrium solution whenever we assume that $\beta_{0} \neq 0$ and $\mu_{2} \neq 0$. To prove this result, we will use the characterization of the exponentially stable semigroups that we can find for instance in the book of Liu and Zheng (1999). In this sense, we recall that whenever the imaginary axis is contained in the resolvent of the generator $\mathcal{A}$ of the semigroup and the condition

$$
\varlimsup_{\lambda \in R,|\lambda| \rightarrow \infty}\left\|(i \lambda \mathcal{I}-\mathcal{A})^{-1}\right\|_{\mathcal{L}(\mathcal{H})}<\infty
$$

holds, the semigroup is exponentially stable.

Then, we prove the following result which states the exponential decay of the energy system.

Theorem 3 Assume that (1)-(2) hold when $2 \tau_{2}>\tau_{1}$, and that $\beta_{0}, \mu_{2} \neq 0$, then the semigroup generated by the operator $\mathcal{A}$ is exponentially stable. That is, there exist two positive constants which are independent of the initial data $N, \eta$, such that

$$
\|U(t)\|_{\mathcal{H}} \leq N \exp (-\eta t)\|U(0)\|_{\mathcal{H}}
$$

for every $U(0) \in \mathcal{H}$.

Proof We shall employ the arguments used in the book of (Liu and Zheng (1999), page 25). In the case that the intersection of the imaginary axis and the spectrum is non-empty, then 
there will exist of real numbers $\lambda_{n}$ with $\lambda_{n} \rightarrow \varpi,\left|\lambda_{n}\right|<|\varpi|$ and a sequence of vectors $U_{n}=\left(u_{n}, v_{n}, \phi_{n}, \varphi_{n}, \theta_{n}, \vartheta_{n}, \zeta_{n}, T_{n}, S_{n}, R_{n}\right)$ in $\mathcal{D}(\mathcal{A})$, and with unit norm, such that

$$
\lim _{n \rightarrow \infty}\left\|\left(i \lambda_{n} \mathcal{I}-\mathcal{A}\right) U_{n}\right\|=0 .
$$

This can be written as

$$
\begin{aligned}
& i \lambda_{n} u_{n}-v_{n} \rightarrow 0 \text { in } W^{1,2}, \\
& i \rho \lambda_{n} v_{n}-\left(\mu u_{n, x x}+\mu_{0} \phi_{n, x}-\beta_{0}\left(\theta_{n, x}+\tau_{1} \vartheta_{n, x}+\frac{\tau_{1}^{2}}{2} \zeta_{n, x}\right)\right) \rightarrow 0 \text { in } L^{2}, \\
& i \lambda_{n} \phi_{n}-\varphi_{n} \rightarrow 0 \text { in } W^{1,2}, \\
& i \rho J \lambda_{n} \varphi_{n}-\left(a_{0} \phi_{n, x x}-\mu_{0} u_{n, x}-\mu_{2}\left(T_{n, x}+\tau_{1} S_{n, x}+\frac{\tau_{1}^{2}}{2} R_{n, x}\right)\right. \\
& \left.\quad+\beta_{1}\left(\theta_{n}+\tau_{1} \vartheta_{n}+\frac{\tau_{1}^{2}}{2} \zeta_{n}\right)-\xi \phi_{n}\right) \rightarrow 0 \text { in } L^{2}, \\
& i \lambda_{n} \theta_{n}-\vartheta_{n} \rightarrow 0 \text { in } W^{1,2}, \\
& i \lambda_{n} \vartheta_{n}-\zeta_{n} \rightarrow 0 \text { in } W^{1,2}, \\
& \frac{i a \tau_{1}^{2} \lambda_{n}}{2} \zeta_{n}+\beta_{0} v_{n, x}+\beta_{1} \varphi_{n}-k\left(\theta_{n, x x}+\tau_{2} \vartheta_{n, x x}\right) \\
& \quad-k_{1}\left(T_{n, x}+\tau_{2} S_{n, x}\right)+a \vartheta_{n}+a \tau_{1} \zeta_{n} \rightarrow 0 \text { in } L^{2}, \\
& i \lambda_{n} T_{n}-S_{n} \rightarrow 0 \text { in } W^{1,2}, \\
& i \lambda_{n} S_{n}-R_{n} \rightarrow 0 \text { in } W^{1,2}, \\
& \frac{i b \tau_{1}^{2} \lambda_{n}}{2} R_{n}+\mu_{2} \varphi_{n, x}-k_{4}\left(T_{n, x x}+\tau_{2} S_{n, x x}\right)+k_{2}\left(T_{n}+\tau_{2} S_{n}\right) \\
& \quad+k_{1}\left(\theta_{n, x}+\tau_{2} \vartheta_{n, x}\right)+a S_{n}+a \tau_{1} R_{n} \rightarrow 0 \text { in } L^{2} .
\end{aligned}
$$

From (6) and the dissipation inequality and the assumptions on the coefficients, we see that $\theta_{n, x}, \vartheta_{n, x}, T_{n, x}$ and $S_{n, x}$ tend to zero in $L^{2}$. Taking the $L^{2}$ inner product of (13) with $\vartheta_{n}$ and (16) with $S_{n}$, respectively, we conclude that $\zeta_{n}$ and $R_{n}$ also tend to zero in $L^{2}$.

Next, in view of (7), we take the $L^{2}$ inner product of (13) with $\lambda_{n}^{-1} u_{n, x}$ to obtain that

$$
\left.i \beta_{0}\left\|u_{n, x}\right\|^{2}+k \lambda_{n}^{-1}\left\langle\theta_{n, x}+\tau_{2} \vartheta_{n, x}, u_{n, x x}\right\rangle+\left.\lambda_{n}^{-1} k\left(\theta_{n, x}+\tau_{2} \vartheta_{n, x}\right) \bar{u}_{n, x}\right|_{0} ^{\pi}\right) \rightarrow 0 .
$$

Hereafter, we use the notation $\|\cdot\|$ and $\langle\cdot, \cdot\rangle$ to denote the $L^{2}$ norm and inner product, respectively. Since $\left\|\lambda_{n}^{-1} u_{n, x x}\right\|$ is bounded (because (13)), we see that $k \lambda_{n}^{-1}\left\langle\theta_{n, x}+\right.$ $\left.\tau_{2} \vartheta_{n, x}, u_{n, x x}\right\rangle$ tends to zero. On the other hand, in view of the Gagliardo-Nirenberg inequality, we know that

$$
\begin{aligned}
& \left|\lambda_{n}\right|^{-1 / 2}\left(\theta_{n, x}+\tau_{2} \vartheta_{n, x}\right)\left\|_{L^{\infty}} \leq K_{1}\left|\lambda_{n}\right|^{-1 / 2}\right\| \theta_{n, x}+\tau_{2} \vartheta_{n, x}\left\|^{1 / 2}\right\| \theta_{n, x x}+\tau_{2} \vartheta_{n, x x} \|^{1 / 2} \\
& \quad+K_{2}\left|\lambda_{n}\right|^{-1 / 2}\left\|\theta_{n, x}+\tau_{2} \vartheta_{n, x}\right\| \rightarrow 0 .
\end{aligned}
$$

In a similar way

$$
\left|\lambda_{n}\right|^{-1 / 2} u_{n, x}\left\|_{L^{\infty}} \leq K_{1}\left|\lambda_{n}\right|^{-1 / 2}\right\| u_{n, x}\left\|^{1 / 2}\right\| u_{n, x x}\left\|^{1 / 2}+K_{2}\left|\lambda_{n}\right|^{-1 / 2}\right\| u_{n, x} \| \rightarrow 0 .
$$

We then conclude that

$$
\left.\lambda_{n}^{-1} k\left(\theta_{n, x}+\tau_{2} \vartheta_{n, x}\right) \bar{u}_{n, x}\right|_{0} ^{\pi} \rightarrow 0 .
$$

Therefore, we obtain that $\left\|u_{n, x}\right\| \rightarrow 0$, and therefore, from (8), we also conclude that $\left\|v_{n}\right\|$ tends to zero. 
A similar argument to the one used to prove that $\left\|u_{n, x}\right\|$ and $\left\|v_{n}\right\|$ tend to zero can be used to prove that $\left\|\phi_{n, x}\right\|$ and $\left\|\varphi_{n}\right\|$ also tend to zero. The only key point is that we now work with (16) in place of (13) and to take $L^{2}$ inner product with $\lambda_{n}^{-1} \phi_{n, x}$ in place of $\lambda_{n}^{-1} u_{n, x}$. However, this contradicts the assumption that $\left\|U_{n}\right\|_{\mathcal{H}}=1$. We conclude that the intersection of the imaginary axis with the spectrum of the operator $\mathcal{A}$ is void.

To conclude the proof of the theorem, we only need to show that condition (5) also holds. Again, we shall use the contradiction argument. It follows that there exist a sequence of real numbers $\lambda_{n}$, such that $\left|\lambda_{n}\right| \rightarrow \infty$ and a sequence of unitary vectors $U_{n}$ in the domain of the operator satisfying (7)- (16). We repeat the arguments used to show that the imaginary axis is contained in the resolvent of the operator to arrive the $\left\|U_{n}\right\| \rightarrow 0$ contradiction. We point out that the only difference is that now $\lambda_{n} \rightarrow \infty$.

\section{Polynomial decay: case of $2 \tau_{2}=\tau_{1}$}

In the case that $2 \tau_{2}=\tau_{1}$, we cannot expect that the decay of the solutions is controlled by a exponential function. In fact, we could adapt to this situation the result obtained in this sense in the case that we do not consider the microtemperatures (see Liu and Quintanilla 2018) . In this section, we prove that the solutions of our problem decay in a polynomial way to the equilibrium solution whenever we assume that $\beta_{0} \neq 0$ and $\mu_{2} \neq 0$. Our result will be a consequence of the characterization proposed by Borichev and Tomilov (2010). In this sense, we recall that whenever the imaginary axis is contained in the resolvent of the generator $\mathcal{A}$ of the semigroup and the condition

$$
\varlimsup_{\lambda \in R,|\lambda| \rightarrow \infty} \lambda^{-2}\left\|(i \lambda \mathcal{I}-\mathcal{A})^{-1}\right\|_{\mathcal{L}(\mathcal{H})}<\infty
$$

holds; the semigroup is polynomially stable. Furthermore, the estimate

$$
\|U(t)\|_{\mathcal{H}} \leq N t^{-1 / 2}\|U(0)\|_{\mathcal{D}(\mathcal{A})}
$$

can be obtained for every $U(0) \in \mathcal{D}(\mathcal{A})$.

To prove that the imaginary axis is contained in the resolvent, we can follow the same way of the previous section. The dissipation inequality implies that $\theta_{n, x}$ and $T_{n, x}$ tend to zero in $L^{2}$. In the case that $\lambda_{n}$ is bounded, we also see that $\vartheta_{n, x}$ and $S_{n, x}$ also tend to zero in $L^{2}$. At this point, we can follow point-by-point the arguments used in the previous section to conclude that the imaginary axis is contained in the resolvent.

Now, we want to prove that the asymptotic condition (17) also holds. Assuming that this is not true, we can find a sequence of vectors

$$
U_{n}=\left(u_{n}, v_{n}, \phi_{n}, \varphi_{n}, \theta_{n}, \vartheta_{n}, \zeta_{n}, T_{n}, S_{n}, R_{n}\right)
$$

in $\mathcal{D}(\mathcal{A})$, and with unit norm, such that

$$
\varlimsup_{\lambda_{n} \in R,\left|\lambda_{n}\right| \rightarrow \infty} \lambda_{n}^{2}\left\|\left(i \lambda_{n} \mathcal{I}-\mathcal{A}\right) U_{n}\right\|_{\mathcal{H}}=0,
$$

that is

$$
\begin{aligned}
& \lambda_{n}^{2}\left(i \lambda_{n} u_{n}-v_{n}\right) \rightarrow 0 \text { in } W^{1,2}, \\
& \lambda_{n}^{2}\left(i \rho \lambda_{n} v_{n}-\left(\mu u_{n, x x}+\mu_{0} \phi_{n, x}-\beta_{0}\left(\theta_{n, x}+\tau_{1} \vartheta_{n, x}+\frac{\tau_{1}^{2}}{2} \zeta_{n, x}\right)\right)\right) \rightarrow 0 \text { in } L^{2}, \\
& \lambda_{n}^{2}\left(i \lambda_{n} \phi_{n}-\varphi_{n}\right) \rightarrow 0 \text { in } W^{1,2},
\end{aligned}
$$




$$
\begin{aligned}
& \lambda_{n}^{2}\left(i \rho J \lambda_{n} \varphi_{n}-\left(a_{0} \phi_{n, x x}-\mu_{0} u_{n, x}-\mu_{2}\left(T_{n, x}+\tau_{1} S_{n, x}+\frac{\tau_{1}^{2}}{2} R_{n, x}\right)\right.\right. \\
& \left.\left.\quad+\beta_{1}\left(\theta_{n}+\tau_{1} \vartheta_{n}+\frac{\tau_{1}^{2}}{2} \zeta_{n}\right)-\xi \phi_{n}\right)\right) \rightarrow 0 \text { in } L^{2}, \\
& \lambda_{n}^{2}\left(i \lambda_{n} \theta_{n}-\vartheta_{n}\right) \rightarrow 0 \text { in } W^{1,2}, \\
& \lambda_{n}^{2}\left(i \lambda_{n} \vartheta_{n}-\zeta_{n}\right) \rightarrow 0 \text { in } W^{1,2}, \\
& \lambda_{n}^{2}\left(\frac{i a \tau_{1}^{2} \lambda_{n}}{2} \zeta_{n}+\beta_{0} v_{n, x}+\beta_{1} \varphi_{n}-k\left(\theta_{n, x x}+\tau_{2} \vartheta_{n, x x}\right)\right. \\
& \left.\quad-k_{1}\left(T_{n, x}+\tau_{2} S_{n, x}\right)+a \vartheta_{n}+a \tau_{1} \zeta_{n}\right) \rightarrow 0 \text { in } L^{2}, \\
& \lambda_{n}^{2}\left(i \lambda_{n} T_{n}-S_{n}\right) \rightarrow 0 \text { in } W^{1,2}, \\
& \lambda_{n}^{2}\left(i \lambda_{n} S_{n}-R_{n}\right) \rightarrow 0 \text { in } W^{1,2}, \\
& \lambda_{n}^{2}\left(\frac{i b \tau_{1}^{2} \lambda_{n}}{2} R_{n}+\mu_{2} \varphi_{n, x}-k_{4}\left(T_{n, x x}+\tau_{2} S_{n, x x}\right)+k_{2}\left(T_{n}+\tau_{2} S_{n}\right)\right. \\
& \left.\quad+k_{1}\left(\theta_{n, x}+\tau_{2} \vartheta_{n, x}\right)+a S_{n}+a \tau_{1} R_{n}\right) \rightarrow 0 \text { in } L^{2} .
\end{aligned}
$$

By (18) and dissipation inequality, we obtain that $\lambda_{n} \theta_{n, x}$ and $\lambda_{n} T_{n, x}$ tend to zero (also in $L^{2}$ ). We can obtain from (23) and (26) that $\vartheta_{n, x}, S_{n, x}$ also tend to zero in $L^{2}$. From this point, we can follow the same argument to the one used in the previous section.

We have proved

Theorem 4 Assume that (1)-(2) hold when $2 \tau_{2}=\tau_{1}$, and that $\beta_{0}, \mu_{2} \neq 0$, then the semigroup generated by the operator $\mathcal{A}$ is polynomially stable. That is, there exists a positive constant $N$, such that

$$
\|U(t)\| \leq N t^{-1 / 2}\|U(0)\|_{\mathcal{D}(\mathcal{A})}
$$

for every $U(0) \in \mathcal{D}(\mathcal{A})$.

\section{Some comments}

In this paper, we have proved several results concerning the decay of the solutions for the porous elasticity when we add dual-phase-lag temperature and microtemperature. To be precise, we have proved that whenever $2 \tau_{2}>\tau_{1}$, the exponential decay of the solutions holds, and when $2 \tau_{2}=\tau_{1}$, the polynomial decay of order 2 of the solution holds. Both results have been obtained under the assumption that $\beta_{0}$ and $\mu_{2}$ are different from zero. In the case when one of this parameters vanishes, we cannot expect the exponential decay and a further study would be needed to clarify the behaviour.

From the previous results, one suspects that the phenomena of the second spectrum (see Ramos et al. 2020) in the case of the porous elasticity can be eliminated when the dual-phaselag thermal effects proposed in the case that $2 \tau_{2}>\tau_{1}$. However, this topic could be the aim of another paper.

In a recent paper, Ramos et al. showed that the solutions of a truncated version of the system of porous elasticity (see Ramos et al. 2020) decay in an exponential way when the porous dissipation is considered. We believe that the inclusion of the thermal effects (also dual-phase-lag) as the only dissipation mechanism brings the system to a similar behaviour. 
Acknowledgements The authors would like to thank the anonymous referees their comments that allow us to improve the paper. The work of R. Quintanilla has been supported by Ministerio de Ciencia, Innovación y Universidades under the research project "Análisis matemático aplicado a la termomecánica” (PID2019105118GB-I00).

Funding Open Access funding provided thanks to the CRUE-CSIC agreement with Springer Nature.

Open Access This article is licensed under a Creative Commons Attribution 4.0 International License, which permits use, sharing, adaptation, distribution and reproduction in any medium or format, as long as you give appropriate credit to the original author(s) and the source, provide a link to the Creative Commons licence, and indicate if changes were made. The images or other third party material in this article are included in the article's Creative Commons licence, unless indicated otherwise in a credit line to the material. If material is not included in the article's Creative Commons licence and your intended use is not permitted by statutory regulation or exceeds the permitted use, you will need to obtain permission directly from the copyright holder. To view a copy of this licence, visit http://creativecommons.org/licenses/by/4.0/.

\section{References}

Bazarra N, Campo M, Fernández JR (2019) A thermoelastic problem with diffusion, microtemperatures, and microconcentrations. Acta Mech 230:31-48

Bazarra N, Copetti MIM, Fernández JR (2021) Quintanilla Numerical analysis of a dual-phase-lag model with microtemperatures. Appl Numer Math 166:1-25

Borgmeyer K, Quintanilla R, Racke R (2014) Phase-lag heat conduction: decay rates for limit problems and well-posedness. J Evol Equat 14:863-884

Borichev A, Tomilov Y (2010) Optimal polynomial decay of functions and operator semigroups. Math Annal 347:455-478

Casas P, Quintanilla R (2005) Exponential stability in thermoelasticity with microtemperatures. Internat J Engrg Sci 43:33-47

Cosserat E, Cosserat F (1909) Théorie des Corps Déformables. Hermannn, Paris

Cowin SC (1985) The viscoelastic behavior of linear elastic materials with voids. J Elasticity 15:185-191

Cowin SC, Nunziato JW (1983) Linear elastic materials with voids. J Elasticity 13:125-147

Eringen C (1999) Microcontinuum Field Theories I: Foundations and Solids. Springer, New York

Feng B, Apalara TA (2019) Optimal decay for a porous elasticity system with memory. J Math Anal Appl 470:1108-1128

Feng B, Yan L, Almeida DS (2020) Stabilizatioin for an inhomogeneous porous-elastic system with temperature and microtemperature. J Appl Math Mech (ZAMM). https://doi.org/10.1002/zamm.202000058

Feng B, Yin M (2019) Decay of solutions for a one-dimensional porous elasticity system with memory: the case of non-equal wave speeds. Math Mech Solids 24:2361-2373

Goodman MA, Cowin SC (1972) A continuum theory for granular materials. Arch Rat Mech Anal 44:249-266

Grot R (1969) Thermodynamics of a continuum with microstructure. Internat J Engrg Sci 7:801-814

Ieşan D (2007) Thermoelasticity of bodies with microstructure and microtemperatures. Internat J Solids Struct 44:8648-8653

Ieşan D (1986) A theory of thermoelastic materials with voids. Acta Mechanica 60:67-89

Ieşan D (2004) Thermoelastic Models of Continua. Springer

Ieşan D, Nappa L (2004) Thermal stresses in plane strain of porous elastic bodies. Meccanica 39:125-138

Ieşan D, Quintanilla R (2000) On a theory of thermoelasticity with microtemperatures. J Therm Stresses 23:195-215

Leseduarte MC, Magaña A, Quintanilla R (2010) On the time decay of solutions in porous-thermo-elasticity of type II. Discrete Cont Dyn Syst B 13:375-391

Liu Z, Quintanilla R (2018) Time decay in dual-phase-lag thermoelasticity:critical case. Comm Pure Appl Anal 17:177-190

Liu Z, Quintanilla R, Wang Y (2017) On the phase-lag equation with spatial dependent. J Math Anal Appl 455:422-438

Liu Z, Quintanilla R, Wang Y (2020) Dual-phase-lag heat conduction with microtemperature, Submitted

Liu Z, Zheng S (1999) Semigroups Associated with Dissipative Systems, Chapman \& Hall/CRC Research Notes in Mathematics, vol 398. Chapman \& Hall/CRC, Boca Raton

Magaña A, Quintanilla R (2006) On the exponential decay of solutions in one-dimensional generalized porousthermo-elasticity. Asymptotic Anal 49:173-187 
Magaña A, Quintanilla R (2007) On the time decay of solutions in porous-elasticity with quasi-static microvoids. J Math Anal Appl 331:617-630

Magaña A, Quintanilla R (2018) Exponential stability in type III thermoelasticity with microtemperatures. Zangew Math Phys 69(5):1291-1298

Magaña A, Quintanilla R (2018) On the existence and uniqueness in phase-lag thermoelasticity. Meccanica 53:125-134

Miranville A, Quintanilla R (2019) Exponential decay in one-dimensional type III thermoelasticity with voids. Appl Math Lett 94:30-37

Miranville A, Quintanilla R (2020) Exponential decay in one-dimensional type II thermoviscoelasticity with voids. J Comput Appl Math 368:112573

Nunziato JW, Cowin SC (1979) A nonlinear theory of elastic materials with voids. Arch Rational Mech Anal 72:175-201

Pamplona PX, Muñoz-Rivera JE, Quintanilla R (2011) On the decay of solutions for porous-elastic systems with history. J Math Anal Appl 379:682-705

Passarella F, Tibullo V, Viccione G (2017) Rayleigh waves in isotropic strongly elliptic thermoelastic materials with microtemperatures. Meccanica 52:3033-3041

Quintanilla R (2003) A condition on the delay parameters in the one-dimensional dual-phase-lag thermoelastic theory. J Therm Stresses 26(7):713-721

Quintanilla R, Racke R (2006) Qualitative aspects in dual-phase-lag thermoelasticity. SIAM J Appl Mathe 66:977-1001

Ramos AJA, Almeida DS, Freitas MM(2020) Dos Santos A new exponential decay result for one-dimensional porous dissipation elasticity from second spectrum viewpoint. Appl Math Lett 101:106061

Riha P (1975) On the theory of heat-conducting micropolar fluids with microtemperatures. Acta Mech 23:1-8

Riha P (1976) On the microcontinuum model of heat conduction in materials with inner structure. Internat J Engrg Sci 14:529-535

Santos ML, Campelo ADS, Almeida Junior DS (2017) On the decay rates of porous elastic systems. J Elasticity 127:79-101

Tzou DY (1995) A unified approach for heat conduction from macro to micro-scales. ASME J Heat Transfer 117:8-16

Publisher's Note Springer Nature remains neutral with regard to jurisdictional claims in published maps and institutional affiliations. 